\title{
ORIGINAL
}

F. D. Fischer · G. A. Zickler · J. Svoboda

\section{Elastic stress-strain analysis of an infinite cylindrical inclusion with eigenstrain}

Received: 13 July 2017 / Accepted: 10 October 2017 / Published online: 8 November 2017

(C) The Author(s) 2017. This article is an open access publication

\begin{abstract}
The ensemble of interstitial atoms as $\mathrm{C}$ attracted to a dislocation is well established as "Cottrell cloud" phenomenon. The deposition of the interstitial atoms in octahedral or tetrahedral positions in a bcc lattice may yield a remarkable internal stress state according to their anisotropic misfit eigenstrains. The stress fields of the atoms may then lead to a significant change in the stress field, e.g., around a dislocation. In such a case, the interstitial atoms are situated near the dislocation core in cylindrical volume elements along the dislocation line. As the occupancy of the sites in each cylinder by interstitial atoms can be considered as constant, also the eigenstrain state tensor is constant in the cylinder. A complete set of analytical expressions for the eigenstress state inside and outside of the cylinder is presented. The resultant stress field is then given by superposition, which allows also the determination of the interaction energy between the Cottrell cloud and the dislocation.
\end{abstract}

Keywords Inclusions · Micro-mechanics · Analytical solution $\cdot$ Linear elasticity $\cdot$ Cylinder

\section{Introduction}

The problem of interaction between dislocations and interstitial atoms as carbon or hydrogen is of increasing interest with respect to a detailed understanding of Cottrell clouds [1], and their kinetics [2]. To calculate the main physical quantity, i.e., the interaction energy between the stress field of a distinct dislocation and of the interstitial atoms, one can use the superposition of the elastic fields generated by the dislocation and by eigenstrains due to deposition of the atoms in interstitial positions. Here the pioneering work by Cochardt et al. [3] can be mentioned, who explained in detail the interaction of a dislocation with a single interstitial atom in the bcc lattice; see also the later Krempasky et al. [4]. There exist three types of anisotropic octahedral positions for interstitial atoms being shorter in one of the three main crystallographic $\langle 100\rangle$ directions in the bcc lattice. Thus, deposition of an atom in one of the octahedral position leads to a remarkably anisotropic eigenstrain in the region of the deposited atom, which can be considered as a spherical inclusion of the volume $\omega$ of a substitutional atom with a given eigenstrain tensor $\varepsilon_{i}^{\omega}$ depending on the type $i, i=1,2,3$, of the octahedral position. This problem of interaction was dealt with also by Friedel [5], Sect. 15.4., in recent papers by Cahn [6,7], Mishin and Cahn [8] and Cai et al. [9]. In most studies, the anisotropic eigenstrain is replaced

F. D. Fischer $(\varangle)$. G. A. Zickler

Institute for Mechanics, Montanuniversität Leoben, Leoben, Austria

E-mail: mechanik@unileoben.ac.at

Tel.: +43 3842/402-4001

J. Svoboda

Institute of Physics of Materials, Academy of Science of the Czech Republic, Brno, Czech Republic

E-mail: svobj@ipm.cz

Tel.: +420/532290407 
by a volume misfit, which is approved for interstitial atoms in fcc lattice or for substitutional atoms in any cubic lattice. In such a case, a rather simple analytical solution for the stress field inside and outside of the inclusion exists. Experimental work, see Wilde et al. [10], and atomistic simulations, Veiga et al. [11], Waseda et al. [12], has shown that an arrangement of interstitial atoms may exist leading to a significant overall stress field stemming from eigenstrains of atoms deposited in the three types of octahedral interstitial positions in the bcc lattice. Since the interaction energy of atoms with the stress field of a distinct dislocation depends significantly on the type of the octahedral interstitial positions, also the local site fraction of atoms in various types of octahedral interstitial positions is significantly different in thermodynamic equilibrium. As a result, the eigenstrains by deposition of interstitial atoms can significantly change the deformation field generated by the dislocation.

The treatment of the interaction with a cloud of interstitial atoms can be, e.g., performed for an edge dislocation, embedded in an infinite elastic lattice, with the dislocation line coinciding with the $z$-axis. Then the cloud of interstitial atoms around the dislocation is independent of $z$ coordinate. The distribution of the interstitial atoms can then be described by a set of cylinders of infinite length along the $z$-axis having small cross sections and possessing in their whole volume constant site fractions of atoms in all three types of interstitial positions. Then the eigenstrain in each cylinder can be calculated and considered as given.

The goal of this paper is to check existing analytical relations for the stress state due to anisotropic eigenstrain state in an infinite cylinder embedded in infinite lattice and to provide a fully analytical solution for the stress field inside and outside of the cylinder. These results will provide the base for a thermodynamic kinetic model treating interaction of interstitial atoms with a moving dislocation.

\section{Problem description}

According to the recent atomistic simulations [11,12], we assume a cylindrical inclusion as a representative volume element with the radius $R$ in the $x-y$-plane normal to the $z$-axis, which may coincide with the dislocation line. We consider a homogeneous eigenstrain state with the in-plane components $\varepsilon_{x}^{\mathrm{eig}}, \varepsilon_{y}^{\mathrm{eig}}, \varepsilon_{x y}^{\mathrm{eig}}$ and the component $\varepsilon_{z}^{\text {eig }}$ in the $z$-direction acting in the inclusion and no eigenstrain in the surrounding matrix. For the sake of completeness, it should be mentioned that the role of eigenstrains, $\varepsilon_{x z}^{\text {eig }}$, $\varepsilon_{y z}^{\text {eig }}$ can be dealt with extra by a simple use of Hooke's law. All the eigenstrain components may have different values.

The cylindrical inclusion is restricted with respect to its expansion in $z$-direction by the surrounding material. Therefore, we assume, as simplest representation of the constraint in $z$-direction, a plane strain state with zero total strain in $z$-direction $\left(\varepsilon_{z} \equiv 0\right)$. A recent atomistic study [8] has shown that the assumption of isotropic material is acceptable for cubic crystals. The isotropic material constants as Young's modulus $E$ and Poisson's ratio $v$ are assumed to be spatially constant in both the inclusion and the surrounding matrix; see Fig. 1.

From pioneering works by Eshelby [13,14], it follows that a homogeneous eigenstrain in an ellipsoidal inclusion induces a homogeneous stress field in the inclusion. If one approximates the cylinder by a needle-like spheroid with $R$ for axes $a$ and $b$ and an infinite length of axis $c$, one may utilize the solutions with normal and shear eigenstrains in Mura's book [15], Chpt. 2 there, or the results worked out in Fischer and Böhm [16]. However, it must be kept in mind that the interstitials interact due to their exterior stress field with the adjacent interstitials and the dislocations. Therefore, the exterior stress field is of immediate relevance. The according mathematical framework in context with the Eshelby concept is rather extensive and somewhat demanding; see also Chpt. 2 in Mura [15] and the treatment by Li et al. [17,18] for inclusions embedded in a finite domain. Our intention to provide easy-to-handle relations for the stress field of a cloud forced us to look for a straightforward solution of our particular problem. Since we have also to consider a shear eigenstrain in the cylinder, the analytical concept by Markenscoff and Dundurs [19], dealing also with a shear eigenstrain in an annulus and the corresponding comment by Shodja and Korshidi [20] concerning the disappearance of the singularities of stresses, motivated us to follow an exact analytical solution.

Since we assume an infinite domain, we expect rather easy-to-handle equations for the stress and deformation state utilizing the Airy function in polar coordinates. Here we refer to the pioneering work by Michell [21] who provided a set of solutions of the biharmonic equation in polar coordinates already in 1899; see also the book by Mal and Singh [22], Sect. 7.5, the book by Barber [23], Sect. 8.4, and the book by Bower [24], Sect. 5.2.3. Such an approach is in accordance with that applied in classical works on circular holes in disks (plates), e.g., by Kirsch [25], more than a century ago, by Bickley [26] and Sen [27]. 


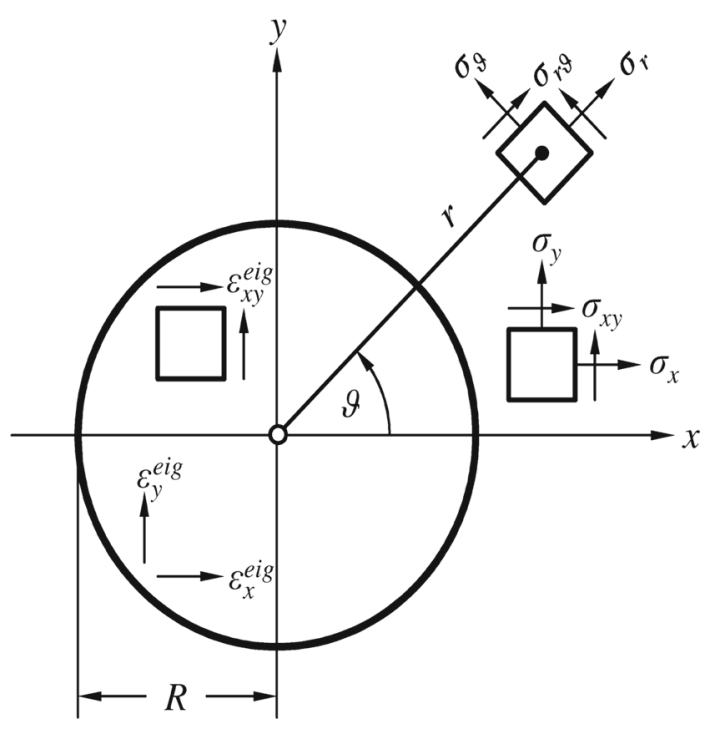

Fig. 1 Sketch of setting

\section{Theory and solution concept}

We introduce cylindrical coordinates $r, \vartheta, z$ and the according displacements $u, v, w$. The kinematic relations between the total strain components $\varepsilon_{r}, \varepsilon_{\vartheta}, \varepsilon_{z}$ and the shear angles $\gamma_{r \vartheta}, \gamma_{r z}, \gamma_{\vartheta z}$ in terms of $u, v, w$ and the equilibrium equations can be taken from any continuum mechanics textbook. Hooke's law links the stress components $\sigma_{r}, \sigma_{\vartheta}, \sigma_{z}, \sigma_{r \vartheta}, \sigma_{r z}, \sigma_{\vartheta z}$ to the elastic strain contributions. Furthermore, we use the following abbreviations

$$
\cos \vartheta=c, \quad \sin \vartheta=s, \quad \cos 2 \vartheta=c_{2}, \quad \sin 2 \vartheta=s_{2} .
$$

\subsection{Solution for the area eigenstrain $\varepsilon^{\text {eig }}$}

Although the solution for this problem is known, for the sake of completeness we start the stress calculation with an in-plane (area) eigenstrain $\varepsilon^{\text {eig }}$ together with an eigenstrain $\varepsilon_{z}^{\text {eig }}$ in $z$-direction keeping in mind that $\varepsilon_{z} \equiv 0$, which yields

$$
\sigma_{z}=-E \varepsilon_{z}^{\mathrm{eig}}+v\left(\sigma_{r}+\sigma_{\vartheta}\right)
$$

Expressing the equilibrium equation only in $u(r)$, since $v=0$ and $w=0$, one finds after some analysis

$$
u=A r+B / r .
$$

The integration constants $A, B$ can be calculated from the two contact conditions

$$
r=R:\left.u\right|_{\mathrm{i}}=\left.u\right|_{\mathrm{o}}, \quad r=R:\left.\sigma_{r}\right|_{\mathrm{i}}=\left.\sigma_{r}\right|_{\mathrm{o}} .
$$

The subscripts " $\mathrm{i}$ " and "o" stand for inside and outside of the inclusion, resp. Finally, we have the following solution for the nonzero stress components as

$$
\begin{array}{cc}
0 \leq r \leq R & \sigma_{r}=\sigma_{\vartheta}=-\frac{E\left(\varepsilon^{\mathrm{eig}}+v \varepsilon_{z}^{\mathrm{eig}}\right)}{2\left(1-v^{2}\right)}, \quad \sigma_{z}=-\frac{E\left(v \varepsilon^{\mathrm{eig}}+\varepsilon_{z}^{\mathrm{eig}}\right)}{\left(1-v^{2}\right)}, \\
r>R & \sigma_{r}=\frac{-E\left(\varepsilon^{\mathrm{eig}}+v \varepsilon_{z}^{\mathrm{eig}}\right)}{2\left(1-v^{2}\right)} \frac{R^{2}}{r^{2}}, \quad \sigma_{\vartheta}=-\sigma_{r}, \quad \sigma_{z}=-E \varepsilon_{z}^{\mathrm{eig}} .
\end{array}
$$

Note that only a deviatoric in-plane stress state exists for $r \geq R$ and $\varepsilon_{z}^{\text {eig }}=0$. This fact is denoted as "Bitter-Crum" theorem; for details, see Fratzl and Penrose [28]. 
3.2 Solution concept for $\varepsilon_{x}^{\text {eig }} \neq 0$ and $\varepsilon_{y}^{\text {eig }} \neq 0$

First we calculate the stress state for $\varepsilon_{x}^{\text {eig }} \neq 0$ only and transform the according eigenstrain tensor in polar coordinates as

$$
\boldsymbol{\varepsilon}_{x}^{\mathrm{eig}}=\frac{\varepsilon_{x}^{\mathrm{eig}}}{2}(\mathbf{I}+\mathbf{P}), \quad \mathbf{P}=\left[\begin{array}{cc}
c_{2} & -s_{2} \\
-s_{2} & -c_{2}
\end{array}\right],
$$

with $\mathbf{I}$ as unit tensor and the abbreviations by Eq. (1). The first part with $\mathbf{I}$ of the r.h.s. of Eq. (6) represents a homogeneous eigenstrain with the solutions of Sect. $3.1 \mathrm{for} \varepsilon^{\text {eig }}=\varepsilon_{x}^{\text {eig }} / 2$ and $\varepsilon_{z}^{\text {eig }}=0$. The second part with $\mathbf{P}$ represents a tensor varying with the angle $2 \vartheta$. Here we select a proper biharmonic Airy stress function $\phi(r, \vartheta)$, inspired by the solution of the "inclusion problem," by Mal and Singh [22], Example 7.5-8, as

$$
\phi=\left(A r^{2}+C r^{4}\right) c_{2} \text { for } 0 \leq r \leq R, \quad \phi=\left(B / r^{2}+D\right) c_{2} \text { for } r \geq R .
$$

We find the nonzero stress components by the following operation, see [21], Sect. 5.2.3 there,

$$
\begin{aligned}
\sigma_{r} & =1 / r \partial \phi / \partial r+1 / r^{2} \partial^{2} \phi / \partial \vartheta^{2}, \\
\sigma_{\vartheta} & =\partial^{2} \phi / \partial r^{2}, \quad \sigma_{r \vartheta}=-\partial / \partial r(1 / r \partial \phi / \partial \vartheta),
\end{aligned}
$$

yielding

$$
\begin{aligned}
0 \leq r \leq R \quad \sigma_{r} & =-2 A c_{2}, \quad \sigma_{\vartheta}=\left(2 A+12 C r^{2}\right) c_{2}, \\
\sigma_{r \vartheta} & =\left(2 A+6 C r^{2}\right) s_{2} ; \\
r \geq R \quad \sigma_{r} & =\left(-6 B / r^{4}-4 D / r^{2}\right) c_{2}, \quad \sigma_{\vartheta}=\left(6 B / r^{4}\right) c_{2}, \\
\sigma_{r \vartheta} & =\left(-6 B / r^{4}-2 D / r^{2}\right) s_{2} .
\end{aligned}
$$

The integration constants $A, B, C, D$ can be calculated from four contact conditions as

$$
r=R:\left.\quad u\right|_{\mathrm{i}}=\left.u\right|_{\mathrm{o}},\left.\vartheta\right|_{\mathrm{i}}=\left.\vartheta\right|_{\mathrm{o}},\left.\quad \sigma_{r}\right|_{\mathrm{i}}=\left.\sigma_{r}\right|_{\mathrm{o}},\left.\quad \sigma_{r \vartheta}\right|_{\mathrm{i}}=\left.\sigma_{r \vartheta}\right|_{\mathrm{o}} .
$$

According to Eshelby [13], the stress state must be homogeneous in the inclusion with the consequence that $C$ must become zero. Furthermore, the contact conditions enforce the calculation of $u(r, \vartheta)$ and $v(r, \vartheta)$ via the integration of the kinematic equations for $\varepsilon_{r}$ and $\varepsilon_{\vartheta}$, combined with Hooke's law (including $\varepsilon_{z}=0$ yielding $\left.\sigma_{z}=v\left(\sigma_{r}+\sigma_{\vartheta}\right)\right)$, as

$$
\begin{aligned}
& E \varepsilon_{r}=E \partial u / \partial r=\sigma_{r}-v \sigma_{\vartheta}-v^{2}\left(\sigma_{r}+\sigma_{\vartheta}\right)+E \varepsilon_{x}^{\mathrm{eig}} c_{2} / 2, \\
& E \varepsilon_{\vartheta}=E(\partial v / \partial \vartheta+u) / r=\left(-v \sigma_{r}+\sigma_{\vartheta}-v^{2}\left(\sigma_{r}+\sigma_{\vartheta}\right)\right)-E \varepsilon_{x}^{\mathrm{eig}} c_{2} / 2
\end{aligned}
$$

The integration of Eqs. (11) involves two functions, namely $f_{u}(\vartheta)$ in $u(r, \vartheta)$ and $g_{v}(r)$ in $v(r, \vartheta)$. These two functions can be found by employing $E \varepsilon_{r \vartheta}$ with $E u$ and $E v$ from above as

$$
E \varepsilon_{r \vartheta}=E\left(\frac{1}{r} \frac{\partial u}{\partial \vartheta}+\frac{\partial v}{\partial r}-\frac{v}{r}\right) / 2=(1+v) \sigma_{r \vartheta}-E \varepsilon_{x}^{\mathrm{eig}} s_{2} / 2
$$

Inserting now $u$ together with $f_{u}(\vartheta)$ and $v$ together with $g_{v}(r)$ in Eq. (12) shows that both $f_{u}(\vartheta)$ and $g_{r}(r)$ can be interpreted as "rigid" body motions and, therefore, can be skipped.

To summarize, the calculation of the coefficients $A, B, C, D$ and, consequently, the stresses makes a lot of algebraic operations necessary, which were left to mathematica (https://www.wolfram.com/mathematica/) but result indeed in $C=0$, see above, and rather simple equations for the stresses. Using the abbreviation $\tilde{E}=E \varepsilon_{x}^{\text {eig }} /\left(8\left(1-v^{2}\right)\right)$ and denoting the stress terms corresponding to $\mathbf{P}$ in Eq. (6) with a subscript "P" yield

$$
\begin{array}{ll}
0 \leq r \leq R: & \sigma_{r}^{\mathbf{P}}=-\tilde{E} c_{2}, \quad \sigma_{\vartheta}^{\mathbf{P}}=\tilde{E} c_{2}, \\
r \geq R: & \sigma_{r \vartheta}^{\mathbf{P}}=\tilde{E} s_{2}, \quad \sigma_{z}^{\mathbf{P}}=0 ; \\
& \sigma_{r}^{\mathbf{P}}=-\tilde{E}\left(4 R^{2} / r^{2}-3 R^{4} / r^{4}\right) c_{2}, \quad \sigma_{\vartheta}^{\mathbf{P}}=-\tilde{E}\left(3 R^{4} / r^{4}\right) c_{2}, \\
\sigma_{r \vartheta}^{\mathbf{P}} & =\tilde{E}\left(3 R^{4} / r^{4}-2 R^{2} / r^{2}\right) s_{2}, \quad \sigma_{z}^{\mathbf{P}}=-4 v \tilde{E} R^{2} / r^{2} .
\end{array}
$$


Let us check now the stress state inside the inclusion with respect to the $x-y$ system. This means that stresses $\sigma_{r}^{\mathbf{P}}, \sigma_{\vartheta}^{\mathbf{P}}, \sigma_{r \vartheta}^{\mathbf{P}}$ must be transformed into the $x-y$ system. The components $\sigma_{x}^{\mathbf{P}}, \sigma_{y}^{\mathbf{P}}, \sigma_{x y}^{\mathbf{P}}$ follow after some algebra

$$
0 \leq r \leq R: \sigma_{x}^{\mathbf{P}}=-\tilde{E}, \quad \sigma_{y}^{\mathbf{P}}=\tilde{E}, \quad \sigma_{x y}^{\mathbf{P}}=0 .
$$

This result is in accordance with the Eshelby [13] theorem, stating that the stress state must be homogeneous in the inclusion for a homogeneous eigenstrain.

The problem of calculating the stress state to an eigenstrain field $\varepsilon_{y}^{\text {eig }}$ only is easily solved by rotation of the total configuration by $\pi / 2$ in relation to the eigenstrain field $\varepsilon_{x}^{\text {eig }}$, yielding, in analogy to Eq. (6),

$$
\boldsymbol{\varepsilon}_{y}^{\mathrm{eig}}=\frac{\varepsilon_{y}^{\mathrm{eig}}}{2}(\mathbf{I}-\mathbf{P})
$$

The superposition of $\boldsymbol{\varepsilon}_{x}^{\text {eig }}$ and $\varepsilon_{y}^{\text {eig }}$ yields for the homogeneous eigenstrain $\varepsilon^{\text {eig }}=\left(\varepsilon_{x}^{\text {eig }}+\varepsilon_{y}^{\text {eig }}\right) / 2$ and for the contribution due to $\mathbf{P}$ the eigenstrain difference $\Delta \varepsilon^{\text {eig }}=\varepsilon_{x}^{\text {eig }}-\varepsilon_{y}^{\text {eig }}$ and with the abbreviations $\tilde{E}^{I}=$ $E\left(\varepsilon^{\mathrm{eig}}+v \varepsilon_{z}^{\mathrm{eig}}\right) / 8\left(1-v^{2}\right) \quad \tilde{E}_{\varepsilon}^{\mathbf{P}}=E \Delta \varepsilon^{\mathrm{eig}} / 8\left(1-v^{2}\right)$,

$$
\begin{aligned}
0 \leq r \leq R: \quad \sigma_{r} & =-4 \tilde{E}^{\mathbf{I}}-\tilde{E}_{\varepsilon}^{\mathbf{P}} c_{2}, \quad \sigma_{\vartheta}=-4 \tilde{E}^{\mathbf{I}}+\tilde{E}_{\varepsilon}^{\mathbf{P}} c_{2}, \\
\sigma_{r \vartheta} & =\tilde{E}_{\varepsilon}^{\mathbf{P}_{2}}, \quad \sigma_{z}=-E \frac{\left(v \varepsilon^{\mathrm{eig}}+\varepsilon_{z}^{\mathrm{eig}}\right)}{\left(1-v^{2}\right)} ; \\
r \geq R: \quad \sigma_{r} & =-4 \tilde{E}^{\mathbf{I}} R^{2} / r^{2}-\tilde{E}_{\varepsilon}^{\mathbf{P}}\left(4 R^{2} / r^{2}-3 R^{4} / r^{4}\right) c_{2}, \quad \sigma_{\vartheta}=4 \tilde{E}^{\mathbf{I}} R^{2} / r^{2}-3 \tilde{E}_{\varepsilon}^{\mathbf{P}}\left(R^{4} / r^{4}\right) c_{2}, \\
\sigma_{r \vartheta} & =\tilde{E}_{\varepsilon}^{\mathbf{P}}\left(3 R^{4} / r^{4}-2 R^{2} / r^{2}\right) s_{2}, \quad \sigma_{z}=-4 v \tilde{E}_{\varepsilon}^{\mathbf{P}}\left(R^{2} / r^{2}\right) c_{2} .
\end{aligned}
$$

\subsection{Solution concept for $\varepsilon_{x y}^{\text {eig }}$}

We complete the results by calculating the stress state according to the shear eigenstrain $\varepsilon_{x y}^{\text {eig }}$ (or the shear angle $\gamma_{x y}^{\text {eig }}=2 \varepsilon_{x y}^{\mathrm{eig}}$ ) only and consider a coordinate system $x^{\prime}-y^{\prime}-z$ rotated by an angle of $\pi / 4$ in relation to the master $x-y-z$ coordinate system with the eigenstrains $\varepsilon_{x^{\prime}}^{\text {eig }}=\varepsilon_{x y}^{\text {eig }}$ and $\varepsilon_{y^{\prime}}^{\text {eig }}=-\varepsilon_{x y}^{\text {eig }}$. Then we can apply the solution concept for $\varepsilon_{x}^{\text {eig }}$ and $\varepsilon_{y}^{\text {eig }}$, however, in the $x^{\prime}-y^{\prime}-z$ coordinate system with $\varepsilon^{\text {eig }}=0, \Delta \varepsilon^{\text {eig }}=\gamma_{x y}^{\text {eig }}$ and $\varepsilon_{z}^{\text {eig }}=0$. Using the abbreviation $\tilde{E}_{\gamma}^{\mathbf{P}}=E \varepsilon_{x y}^{\mathrm{eig}} / 4\left(1-v^{2}\right)$ and noting that $\vartheta^{\prime}=\vartheta-\pi / 4$, the relations in the master coordinate system read as
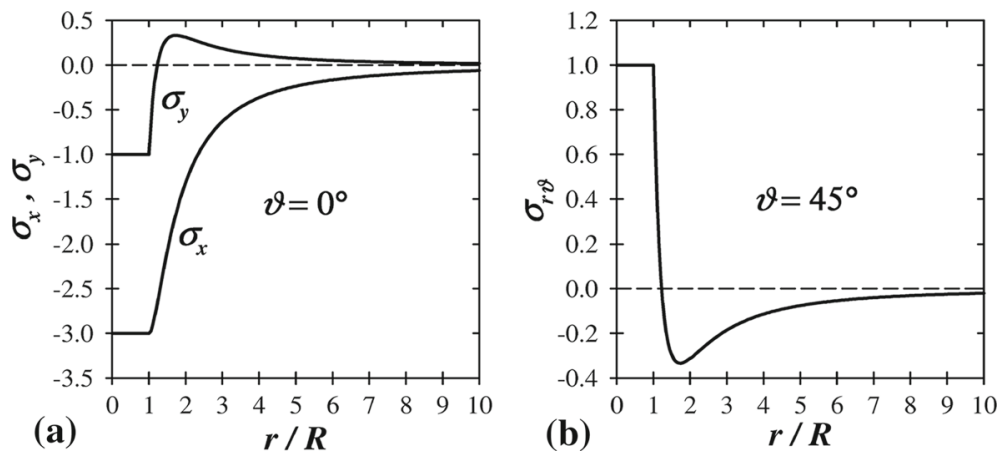

Fig. 2 Eigenstrain $\varepsilon_{x}^{\text {eig }}, E \varepsilon_{x}^{\text {eig }}=8\left(1-v^{2}\right)$; (a) stress state $\sigma_{x}, \sigma_{y}$ along $x$-axis, (b) shear stress $\sigma_{r \vartheta}$ along $r$ at $\vartheta=45^{\circ}$ 

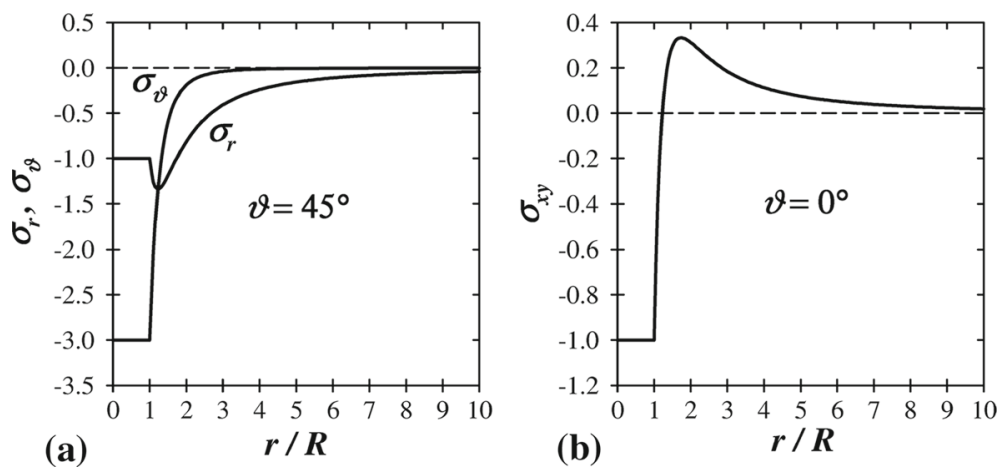

Fig. 3 Eigenstrain $\varepsilon_{x y}^{\mathrm{eig}}, E \varepsilon_{x y}^{\mathrm{eig}}=4\left(1-v^{2}\right)$; (a) normal stresses $\sigma_{r}, \sigma_{\vartheta}$ along $r$ at $\vartheta=45^{\circ}$, (b) shear stress $\sigma_{x y}$ along $x$-axis

$$
\begin{aligned}
0 \leq r \leq R \quad \sigma_{r} & =-\tilde{E}_{\gamma}^{\mathbf{P}} s_{2}, \quad \sigma_{\vartheta}=\tilde{E}_{\gamma}^{\mathbf{P}} s_{2}, \\
\sigma_{r \vartheta} & =-\tilde{E}_{\gamma}^{\mathbf{P}} c_{2}, \quad \sigma_{z}=0 ; \\
R<r \quad \sigma_{r} & =-\tilde{E}_{\gamma}^{\mathbf{P}}\left(4 R^{2} / r^{2}-3 R^{4} / r^{4}\right) s_{2}, \quad \sigma_{\vartheta}=-3 \tilde{E}_{\gamma}^{\mathbf{P}}\left(R^{4} / r^{4}\right) s_{2}, \\
\sigma_{r \vartheta} & =-\tilde{E}_{\gamma}^{\mathbf{P}}\left(3 R^{4} / r^{4}-2 R^{2} / r^{2}\right) c_{2}, \quad \sigma_{z}=-4 v \tilde{E}_{\gamma}^{\mathbf{P}}\left(R^{2} / r^{2}\right) s_{2} .
\end{aligned}
$$

\section{Representative examples and discussion}

We demonstrate two examples in a dimension-free form. Assuming $E \varepsilon_{x}^{\text {eig }}=8\left(1-v^{2}\right)$, we demonstrate $\sigma_{x}$ and $\sigma_{y}$ along the $x$-axis in Fig. 2 a and $\sigma_{r \vartheta}$ along the radius $r$ for $\vartheta=45^{\circ}$ in Fig. 2b. Assuming $E \varepsilon_{x y}^{\text {eig }}=4\left(1-v^{2}\right)$ we demonstrate $\sigma_{r}$ and $\sigma_{\vartheta}$ along the radius $r$ for $\vartheta=45^{\circ}$ in Fig. 3a and $\sigma_{x y}$ along the $x$-axis in Fig. 3b. All curves are checked by a finite element study with ABAQUS (http://www.3ds.com/de/produkte-und-services/ simulia/produkte/abaqus/).

It is interesting to note that the stresses outside of the inclusion, dominated by a $(R / r)^{2}$ term, decay to nearly zero over a remarkably long distance of approximately $10 R$.

For practical application of the results, we present for each kind of eigenstrain (i.e., $\varepsilon_{x}^{\text {eig }}, \varepsilon_{y}^{\text {eig }}, \varepsilon_{x y}^{\text {eig }}, \varepsilon_{z}^{\text {eig }}$ ) the stress fields in a Cartesian coordinate system in "Appendix."

Acknowledgements Open access funding provided by Montanuniversität Leoben. Financial support by the Austrian Federal Government (in particular from the Bundesministerium für Verkehr, Innovation and Technologie and the Bundesministerium für Wirtschaft und Arbeit) and the Styrian Provincial Government, represented by Österreichische Forschungsförderungsgesellschaft $\mathrm{mbH}$ and by Steirische Wirtschaftsförderungsgesellschaft $\mathrm{mbH}$, within the research activities of the K2 Competence Centre on "Integrated Research in Materials, Processing and Product Engineering," operated by the Materials Center Leoben Forschung $\mathrm{GmbH}$ in the framework of the Austrian COMET Competence Centre Programme, Projects A1.23 and A2.32, is gratefully acknowledged. J.S. gratefully acknowledges the financial support by the Czech Science Foundation in the frame of the Project 17-01641S.

Open Access This article is distributed under the terms of the Creative Commons Attribution 4.0 International License (http:// creativecommons.org/licenses/by/4.0/), which permits unrestricted use, distribution, and reproduction in any medium, provided you give appropriate credit to the original author(s) and the source, provide a link to the Creative Commons license, and indicate if changes were made.

\section{Appendix: Eigenstress fields in a Cartesian coordinate system}

With the abbreviations in Eq. (1), we have

$$
\begin{gathered}
\sigma_{x}=c^{2} \sigma_{r}+s^{2} \sigma_{\vartheta}-s_{2} \sigma_{r \vartheta}, \quad \sigma_{y}=s^{2} \sigma_{r}+c^{2} \sigma_{\vartheta}-s_{2} \sigma_{r \vartheta} \\
\sigma_{x y}=s c\left(\sigma_{r}-\sigma_{\vartheta}\right)+c_{2} \sigma_{r \vartheta}, \quad \sigma_{z}=-E \varepsilon_{z}^{\mathrm{eig}}+v\left(\sigma_{r}+\sigma_{\vartheta}\right),
\end{gathered}
$$

with $c=x / r, s=y / r$ and $r^{2}=x^{2}+y^{2}$ and $\bar{x}=x / R, \bar{y}=y / R$ and $\bar{r}=r / R$. 
Expressing the relations for the individual cases of eigenstrain yields:

$$
\varepsilon_{x}^{\text {eig }} \neq 0
$$

Inside

$$
\sigma_{x}=-\frac{3 E \varepsilon_{x}^{\mathrm{eig}}}{8\left(1-v^{2}\right)}, \quad \sigma_{y}=-\frac{E \varepsilon_{x}^{\mathrm{eig}}}{8\left(1-v^{2}\right)}, \quad \sigma_{z}=-\frac{E v \varepsilon_{x}^{\mathrm{eig}}}{2\left(1-v^{2}\right)} .
$$

Outside

$$
\begin{aligned}
& \sigma_{x}=-\frac{E \varepsilon_{x}^{\mathrm{eig}}\left[2 \bar{r}^{4}\left(\bar{x}^{2}-\bar{y}^{2}\right)+4 \bar{r}^{2} \bar{x}^{2}\left(\bar{x}^{2}-3 \bar{y}^{2}\right)-3\left(\bar{x}^{4}-6 \bar{x}^{2} \bar{y}^{2}+\bar{y}^{4}\right)\right]}{8\left(1-v^{2}\right) \bar{r}^{8}}, \\
& \sigma_{y}=\frac{E \varepsilon_{x}^{\mathrm{eig}}\left[2 \bar{r}^{4}\left(\bar{x}^{2}-\bar{y}^{2}\right)-4 \bar{r}^{2} \bar{y}^{2}\left(3 \bar{x}^{2}-\bar{y}^{2}\right)-3\left(\bar{x}^{4}-6 \bar{x}^{2} \bar{y}^{2}+\bar{y}^{4}\right)\right]}{8\left(1-v^{2}\right) \bar{r}^{8}}, \\
& \sigma_{x y}=-\frac{E \varepsilon_{x}^{\mathrm{eig}} \bar{x} \bar{y}\left[\bar{r}^{4}+\left(2 \bar{r}^{2}-3\right)\left(\bar{x}^{2}-\bar{y}^{2}\right)\right]}{2\left(1-v^{2}\right) \bar{r}^{8}}, \quad \sigma_{z}=-\frac{E v \varepsilon_{x}^{\mathrm{eig}}\left(\bar{x}^{2}-\bar{y}^{2}\right)}{2\left(1-v^{2}\right) \bar{r}^{4}} . \\
& \varepsilon_{y}^{\mathrm{eig}} \neq 0
\end{aligned}
$$

Inside

$$
\sigma_{x}=-\frac{E \varepsilon_{y}^{\mathrm{eig}}}{8\left(1-v^{2}\right)}, \quad \sigma_{y}=-\frac{3 E \varepsilon_{y}^{\mathrm{eig}}}{8\left(1-v^{2}\right)}, \quad \sigma_{z}=-\frac{E v \varepsilon_{y}^{\mathrm{eig}}}{2\left(1-v^{2}\right)}
$$

Outside

$$
\begin{gathered}
\sigma_{x}=-\frac{E \varepsilon_{y}^{\mathrm{eig}}\left[2 \bar{r}^{4}\left(\bar{x}^{2}-\bar{y}^{2}\right)-4 \bar{r}^{2} \bar{x}^{2}\left(\bar{x}^{2}-3 \bar{y}^{2}\right)+3\left(\bar{x}^{4}-6 \bar{x}^{2} \bar{y}^{2}+\bar{y}^{4}\right)\right]}{8\left(1-v^{2}\right) \bar{r}^{8}}, \\
\sigma_{y}=\frac{E \varepsilon_{y}^{\mathrm{eig}}\left[2 \bar{r}^{4}\left(\bar{x}^{2}-\bar{y}^{2}\right)+4 \bar{r}^{2} \bar{y}^{2}\left(3 \bar{x}^{2}-\bar{y}^{2}\right)+3\left(\bar{x}^{4}-6 \bar{x}^{2} \bar{y}^{2}+\bar{y}^{4}\right)\right]}{8\left(1-v^{2}\right) \bar{r}^{8}}, \\
\sigma_{x y}=-\frac{E \varepsilon_{y}^{\mathrm{eig}} \bar{x} \bar{y}\left[\bar{r}^{4}-\left(2 \bar{r}^{2}-3\right)\left(\bar{x}^{2}-\bar{y}^{2}\right)\right]}{2\left(1-v^{2}\right) \bar{r}^{8}}, \quad \sigma_{z}=-\frac{E v \varepsilon_{y}^{\mathrm{eig}}\left(\bar{x}^{2}-\bar{y}^{2}\right)}{2\left(1-v^{2}\right) \bar{r}^{4}} . \\
\varepsilon_{x y}^{\mathrm{eig}} \neq 0
\end{gathered}
$$

Inside

$$
\sigma_{x}=\sigma_{y}=0, \quad \sigma_{x y}=-\frac{E \varepsilon_{x y}^{\mathrm{eig}}}{4\left(1-v^{2}\right)},
$$

Outside

$$
\begin{gathered}
\sigma_{x}=-\frac{E \varepsilon_{x y}^{\mathrm{eig}} \bar{x} \bar{y}\left[\bar{r}^{2}\left(3 \bar{x}^{2}-\bar{y}^{2}\right)-3\left(\bar{x}^{2}-\bar{y}^{2}\right)\right]}{\left(1-v^{2}\right) \bar{r}^{8}}, \quad \sigma_{y}=\frac{E \varepsilon_{x y}^{\mathrm{eig}} \bar{x} \bar{y}\left[\bar{r}^{2}\left(\bar{x}^{2}-3 \bar{y}^{2}\right)-3\left(\bar{x}^{2}-\bar{y}^{2}\right)\right]}{\left(1-v^{2}\right) \bar{r}^{8}}, \\
\sigma_{x y}=\frac{E \varepsilon_{x y}^{\mathrm{eig}}\left(2 \bar{r}^{2}-3\right)\left(\bar{x}^{4}-6 \bar{x}^{2} \bar{y}^{2}+\bar{y}^{4}\right)}{4\left(1-v^{2}\right) \bar{r}^{8}}, \quad \sigma_{z}=-\frac{2 E v \varepsilon_{x y}^{\mathrm{eig}}(\bar{x} \bar{y})}{\left(1-v^{2}\right) \bar{r}^{4}} . \\
\varepsilon_{z}^{\text {eig }} \neq 0
\end{gathered}
$$

Inside

$$
\sigma_{x}=-\frac{E v \varepsilon_{z}^{\mathrm{eig}}}{2\left(1-v^{2}\right)}, \quad \sigma_{y}=-\frac{E v \varepsilon_{z}^{\mathrm{eig}}}{2\left(1-v^{2}\right)}, \quad \sigma_{z}=-\frac{E \varepsilon_{z}^{\mathrm{eig}}}{\left(1-v^{2}\right)} .
$$


Outside

$$
\begin{aligned}
\sigma_{x} & =-\frac{E v \varepsilon_{z}^{\mathrm{eig}}\left(\bar{x}^{2}-\bar{y}^{2}\right)}{2\left(1-v^{2}\right) \bar{r}^{4}}, \quad \sigma_{y}=\frac{E v \varepsilon_{z}^{\mathrm{eig}}\left(\bar{x}^{2}-\bar{y}^{2}\right)}{2\left(1-v^{2}\right) \bar{r}^{4}}, \\
\sigma_{x y} & =-\frac{E v \varepsilon_{z}^{\mathrm{eig}} \bar{x} \bar{y}}{\left(1-v^{2}\right) \bar{r}^{4}}, \quad \sigma_{z}=0 .
\end{aligned}
$$

\section{References}

1. Cottrell, A.H., Bilby, B.A.: Dislocation theory on yielding and strain ageing in iron. Proc. Phys. Soc. Lond. Sect. A 62, 49-62 (1949)

2. Svoboda, J., Zickler, G.A., Kozeschnik, E., Fischer, F.D.: Kinetics of interstitials segregation in Cottrell atmospheres and grain boundaries. Philos. Mag. Lett. 95, 458-465 (2015)

3. Cochardt, A.W., Schoeck, G., Wiedersich, H.: Interaction between dislocations and interstitial atoms in body-centered cubic metals. Acta Metall. 3, 533-537 (1955)

4. Krempasky, C., Liedl, U., Werner, E.A.: A note on the diffusion of carbon atoms to dislocations. Comput. Mater. Sci. 38, 90-97 (2006)

5. Friedel, J.: Dislocations, Pergamon Student Editions, vol. 3. Pergamon Press, Oxford (1964)

6. Cahn, J.W.: Thermodynamic aspects of Cottrell atmospheres. Philos. Mag. 93, 3741-3746 (2013)

7. Cahn, J.W.: Reprise: partial chemical strain dislocations and their role in pinning dislocations to their atmospheres. Philos. Mag. 94, 3170-3176 (2014)

8. Mishin, Y., Cahn, J.W.: Thermodynamics of Cottrell atmospheres tested by atomistic simulations. Acta Mater. 117, 197-206 (2016)

9. Cai, W., Sills, R.B., Barnett, D.M., Nix, W.D.: Modeling a distribution of point defects as misfitting inclusions in stressed solids. J. Mech. Phys. Solids 66, 154-171 (2014)

10. Wilde, J., Cerezo, A., Smith, G.D.W.: Three-dimensional atomic-scale mapping of a Cottrell atmosphere around a dislocation in iron. Scripta Mater. 43, 39-48 (2000)

11. Veiga, R.G.A., Perez, M., Becquart, C.S., Comain, C.: Atomistic modeling of carbon Cottrell atmospheres in bcc iron. J. Phys. Condens. Matter 25, 025401 (2013). (7 pp)

12. Waseda, O., Veiga, R.G.A., Morthomas, J., Chantrenne, P., Becquart, C.S., Ribeiro, F., Jelea, A., Goldenstein, H., Perez, M.: Formation of carbon Cottrell atmospheres and their effect on the stress field around an edge dislocation. Scripta Mater. 129, 16-19 (2017)

13. Eshelby, J.D.: The determination of the elastic field of an inclusion, and related problems. Proc. R. Soc. Lond. A 241, 376-396 (1957)

14. Eshelby, J.D.: The elastic field outside an ellipsoidal inclusion. Proc. R. Soc. Lond. A 252, 561-569 (1959)

15. Mura, T.: Micromechanics of Defects in Solids, 2nd edn. Martinus Nijhoff Publishers, Dordrecht (1987)

16. Fischer, F.D., Böhm, H.J.: On the role of transformation eigenstrain in the growth or shrinkage of a spheroidal inclusion, with a general eigenstrain state. Acta Mater. 54(151-156), $55(2006,2007)$

17. Li, S., Sauer, R., Wang, G.: A cicular inclusion in a finite domain I. The Dirichlet-Eshelby problem. Acta Mech. 179, 67-90 (2005)

18. Wang, G., Li, S., Sauer, R.: A circular inclusion in a finite domain II. The Neumann-Eshelby problem. Acta Mech. 179, 91-110 (2005)

19. Markenscoff, X., Dundurs, J.: Annular inhomogeneities with eigenstrain and interphase modeling. J. Mech. Phys. Solids 64, 468-482 (2014)

20. Shodja, H.M., Khorshidi, A.: Comment on "Annular inhomogeneities with eigenstrain and interphase modeling [2014, J. Mech. Phys. Solids 64, 468-482]”. J. Mech. Phys. Solids 73, 1-2 (2014)

21. Michell, J.H.: On the direct determination of stress in elastic solid, with application to the theory of plates. Proc. Lond. Math. Soc. 31, 100-124 (1899)

22. Mal, A.K., Singh, S.J.: Deformation of Elastic Solids. Prentice-Hall, New Jersey (1991)

23. Barber, J.R.: Elasticity, 1st edn. Kluwer Academic Publishers, Dordrecht (1992)

24. Bower, A.F.: Applied Mechanics of Solids. CRC Press, Boca Raton (2009)

25. Kirsch, G.: Die Theorie der Elastizität und die Bedürfnisse der Festigkeitslehre. Z. VDI 42, 797-807 (1898). (in German)

26. Bickley, W.G.: The distribution of stress round a circular hole in a plate. Philos. Trans. R. Soc. Lond. 227, 383-415 (1928)

27. Sen, B.: Problems of thin plates with circular holes. Bull. Calcutta Math. Soc. 37, 37-42 (1945)

28. Fratzl, P., Penrose, O., Lebowitz, J.L.: Modelling of phase separation in alloys with coherent elastic misfit. J. Stat. Phys. 95, 1429-1503 (1999) 